\title{
HIGH-VELOCITY ABSORPTION COMPONENTS TOWARD THE LMC
}

\author{
G. Vladilo ${ }^{1}$, P. Molaro ${ }^{1}$, S. Monai $^{2}$, and M. Centurion ${ }^{1}$ \\ 1 Osservatorio Astronomico di Trieste \\ ${ }^{2}$ Dipartimento di Astronomia - Università di Trieste
}

\section{ABSTRACT}

High-resolution $\left(\lambda / \delta \lambda \simeq 6 \times 10^{4}\right)$ spectra of R136 (the central object of 30 Dor) and of 2 LMC supergiants obtained at ESO with the 1.4-m telescope have been analysed in order to study the high-velocity absorption components at $v_{L S R} \simeq+60 \mathrm{~km} \mathrm{~s}^{-1}$ and $\simeq+130 \mathrm{~km} \mathrm{~s}^{-1}$ first seen in satellite ultraviolet spectra in the general direction of the LMC. Our data have been integrated with previous literature results of SN1987a and of other 4 LMC supergiants obtained from spectra of similar spectral resolution.

At least 6 (possibly 9) interstellar components are found in the range $+40 \mathrm{~km} \mathrm{~s}^{-1} \leq v_{L S R} \leq$ $+150 \mathrm{~km} \mathrm{~s}^{-1}$, suggesting that the IUE features at $\simeq+60 \mathrm{~km} \mathrm{~s}^{-1}$ and $\simeq+130 \mathrm{~km} \mathrm{~s}^{-1}$ are in fact splitted in several components when observed at higher resolution. Two components originally discovered toward SN1987a $\left(v_{L S R} \simeq+42 \mathrm{~km} \mathrm{~s}^{-1}\right.$ and $\left.+107 \mathrm{~km} \mathrm{~s}^{-1}\right)$, are detected for the first time in the CaII interstellar spectrum of R136. A component at $v_{L S R} \simeq+49 \mathrm{~km} \mathrm{~s}^{-1}$ shows significant variations both in CaII and in $\mathrm{NaI}$ along the different lines of sight. A relatively broad component at $v_{L S R} \simeq+58 \mathrm{~km} \mathrm{~s}^{-1}$ is present in CaII toward R136, but is generally undetected in $\mathrm{NaI}$ in the other lines of sight. Also the components in the range $+100 \mathrm{~km} \mathrm{~s}^{-1} \leq v_{L S R} \leq+150$ $\mathrm{km} \mathrm{s}^{-1}$ are detected in CaII, but not in NaI. Two weak absorptions, detected at $+125 \mathrm{~km} \mathrm{~s}^{-1}$ and $+141 \mathrm{~km} \mathrm{~s}^{-1}$ toward $\mathrm{R} 136$, may be associated with the $\simeq+130 \mathrm{~km} \mathrm{~s}^{-1}$ component characteristic of the ultraviolet spectra.

The almost perfect velocity coincidence of the $+107 \mathrm{~km} \mathrm{~s}^{-1},+42 \mathrm{~km} \mathrm{~s}^{-1},+49 \mathrm{~km} \mathrm{~s}^{-1}$ and $+57 \mathrm{~km} \mathrm{~s}^{-1}$ components along different lines of sight suggests a common origin of the absorbing gas and a location close to our Galaxy. In spite of the low detection limit in our spectra $\left(\mathrm{W}_{\lambda} \simeq 5\right.$ $\mathrm{m} \AA$ ), the lack of detection of $\mathrm{NaI}$ is compatible with the presence of foreground gas clouds with hydrogen column density $\mathrm{N}(\mathrm{HI}) \leq 2 \times 10^{19} \mathrm{~cm}^{-2}$, or even greater. The fact that we can detect more easily CaII absorptions than the corresponding $\mathrm{NaI}$ absorptions indicates a very low value of CaII depletion.

\section{INTRODUCTION}

During the last decade the interstellar medium in the direction of the Large Magellanic Cloud (LMC) has been investigated by means of absorption line spectra in the ultraviolet (Savage and de Boer, 1979, 1981; FWHM $\simeq 20-25 \mathrm{~km} \mathrm{~s}^{-1}$ ) and in the optical (Blades and Meaburn, 1980; Songaila, 1981; Songaila et al., 1986 and ref.s therein; FWHM $\left.\simeq 8-15 \mathrm{~km} \mathrm{~s}^{-1}\right)$. The IUE data lead to the discovery of absorption features near $+60 \mathrm{~km} \mathrm{~s}^{-1}$ and $+130 \mathrm{~km} \mathrm{~s}^{-1}$, interpreted by Savage and de Boer (1981) as produced in an extended gaseous halo, co-rotating with the disk of the Galaxy. Songaila at al. (1986), from the analysis of CaII spectra of 30 stars in the LMC, suggested that these features may arise inside the LMC.

In the past few years, improvements in the ground-based technology have allowed to perform observations at very high spectral resolution (FWHM $\simeq 5 \mathrm{~km} \mathrm{~s}^{-1}$ ) and signal-to-noise (S/N $\simeq$ 50) toward some LMC supergiants (Ferlet et al., 1985a; Molaro et al., 1989). The explosion of SN1987a has provided a unique background source toward which an extremely rich interstellar 
spectrum has been observed in great detail (Vidal-Madjar et al., 1987; FWHM $\simeq 3 \mathrm{~km} \mathrm{~s}^{-1} ; \mathrm{S} / \mathrm{N}$ $\geq 100$ ). In this communication we present new Call spectra of R136 (the central object of the 30 Dor nebula) and NaI spectra of two LMC supergiants (Sk-67 44 and Sk-67 201). Our data are compared with previous optical observations towards other lines of sight characterized by a similar quality ( $\mathrm{FWHM} \simeq 5 \mathrm{~km} \mathrm{~s}^{-1} ; \mathrm{S} / \mathrm{N} \simeq 50$ ), in order to study the high-velocity components detected by Savage and de Boer in the ultraviolet. The relevant data for the target stars are shown in Table I.

\section{OBSERVATIONS AND DATA REDUCTION}

The observations were performed in April 1988 with the Coudè Echelle Spectrograph (CES) fed by the 1.4-m telescope at the European Southern Observatory (ESO), La Silla. The spectra were obtained using a CCD detector with the short camera of the CES at $\lambda / \delta \lambda \simeq 6 \times 10^{4}$. Due to the fall of sensitivity of the CCD toward the blue spectral region, only the brightest object, R136, was observed in $\mathrm{CaII}$. Sk- $67^{\circ} 44$ and $\mathrm{Sk}-67^{\circ} 201$ were observed in NaI. A template star ( $\alpha \mathrm{Pic}$ ) was used as a reference for correction of the telluric contamination in the $\mathrm{NaI}$ spectral region. The spectra were calibrated using standard commands of IHAP, the ESO image processing system. The resulting minimum detectable equivalent width in our spectra ( $3 \sigma$ level) is of about $5 \mathrm{~m} \AA$. After normalization of the spectra to the stellar continuum, the interstellar absorptions were measured using multiple component gaussian fits. The wavelength scale was converted to LSR radial velocity scale $\left(v_{L S R}=v_{h e l}-15.2 \mathrm{~km} \mathrm{~s}^{-1}\right)$, with a final error lower than $\pm 1.0 \mathrm{~km} \mathrm{~s}^{-1}$. In Fig.s 1 and 2 we show the CaII spectrum of R136 and the NaI spectrum of Sk-67 44 , respectively.

\section{HIGH-VELOCITY ABSORPTION COMPONENTS}

In Table II we list a summary of the data in the range $+40 \mathrm{~km} \mathrm{~s}^{-1} \leq v_{L S R} \leq+150 \mathrm{~km} \mathrm{~s}^{-1}$. For each component detected in at least one line of sight we give the LSR radial velocity, $v_{L S R}$ $\left(\mathrm{km} \mathrm{s}^{-1}\right)$, and the equivalent width, $W_{\lambda}(\mathrm{m} \AA)$. Only optical spectra with spectral resolution $\lambda / \delta \lambda \geq 5 \times 10^{4}$ have been considered. Data from previous literature works are indicated in the Table. The comparison in the NaI region has been performed along 7 lines of sight, while in the CaII region only between R136 and SN1987a.

In the range $+40 \mathrm{~km} \mathrm{~s}^{-1} \leq v_{L S R} \leq+60 \mathrm{~km} \mathrm{~s}^{-1}$ there are at least 3 interstellar components detected in CaII toward the supernova and in our spectrum of R136. This suggests that the absorption originally discovered in the ultraviolet near $+60 \mathrm{~km} \mathrm{~s}^{-1}$ is in fact the result of a blend of different components.

A weak CaII absorption at $+42 \mathrm{~km} \mathrm{~s}^{-1}$ is detected in R136, which could be associated to a CaII feature at the same velocity observed in the supernova. This component is not observed in $\mathrm{NaI}$ in any line of sight.

The component around $+49 \mathrm{~km} \mathrm{~s}^{-1}$ is detected in 4 (possibly 5) of the 7 lines of sight investigated in NaI. The strong CaII feature visible at the same velocity in Sn1987a is almost absent in R136. This component shows strong variations along the different lines of sight, both in $\mathrm{NaI}$ and in CaIl. An indication for the location of the $+49 \mathrm{~km} \mathrm{~s}^{-1}$ component comes from the NaI spectra of Sk- $69^{\circ} 220$ and Sk- $69^{\circ} 221$. These stars are LMC members, but do not show any significant NaI absorption at the LMC systemic velocity, suggesting that they are situated in front of the bulk of the LMC gas. Since the $+49 \mathrm{~km} \mathrm{~s}^{-1}$ feature is present in their spectra, the corresponding cloud cannot lie behind the bulk of the LMC gas, and should be associated to the halo of our Galaxy, or to a halo surrounding the LMC.

A relatively broad CaII component is seen near $v_{L S R} \simeq+58 \mathrm{~km} \mathrm{~s}^{-1}$ toward SN1987a and $\mathrm{R} 136$. In the $\mathrm{NaI}$ region the same component is marginally detected in the supernova, is present 
TABLE I

LMC bright sources observed at high spectral resolution (FWHM $\leq 5 \mathrm{~km} \mathrm{~s}^{-1}$ ) in the optical

\begin{tabular}{|c|c|c|c|c|}
\hline Star & $\mathrm{m}_{V}$ & $\begin{array}{l}\text { Spectral } \\
\text { type }\end{array}$ & $\begin{array}{l}\text { R.A. } \\
(1950)\end{array}$ & $\begin{array}{l}\text { Decl. } \\
(1950)\end{array}$ \\
\hline SN1987a & & & $5^{h} 36^{m}$ & $-69^{\circ} 19^{\prime}$ \\
\hline $\mathrm{R} 136$ & 9.50 & WR & $5^{h} 39^{m}$ & $-69^{\circ} 07^{\prime}$ \\
\hline Sk- $67^{\circ} 44$ & 9.21 & A4Ia & $5^{h} 06^{m}$ & $-67^{\circ} 57^{\prime}$ \\
\hline Sk- $67^{\circ} 201$ & 9.90 & AOIae & $5^{h} 34^{m}$ & $-67^{\circ} 03^{\prime}$ \\
\hline Sk- $69^{\circ} 203$ & 12.29 & B0.5 & $5^{h} 36^{m}$ & $-69^{\circ} 16^{\prime}$ \\
\hline Sk- $69^{\circ} 211$ & 10.36 & B8 I & $5^{h} 37^{m}$ & $-69^{\circ} 26^{\prime}$ \\
\hline Sk- $69^{\circ} 220$ & 11.40 & Bep & $5^{h} 37^{m}$ & $-69^{\circ} 32^{\prime}$ \\
\hline Sk- $69^{\circ} 221$ & 10.63 & B1Iab & $5^{h} 37^{m}$ & $-69^{\circ} 30^{\prime}$ \\
\hline
\end{tabular}

TABLE II

High-velocity interstellar components toward the targets of Table I

NaI D2

\begin{tabular}{|c|c|c|c|c|c|c|c|c|c|c|c|c|c|}
\hline \multicolumn{2}{|c|}{ SN1987a } & \multicolumn{2}{|c|}{ Sk- $67^{\circ} 44^{2}$} & \multicolumn{2}{|c|}{ Sk- $67^{\circ} 201^{2}$} & \multicolumn{2}{|c|}{ Sk- $69^{\circ} 203^{3}$} & \multicolumn{2}{|c|}{ Sk- $69^{\circ} 211^{3}$} & \multicolumn{2}{|c|}{ Sk- $69^{\circ} 220^{4}$} & \multicolumn{2}{|c|}{ Sk- $69^{\circ} 221^{4}$} \\
\hline$v_{L S R}$ & $W_{\lambda}$ & $v_{L S R}$ & $W_{\lambda}$ & $v_{L S R}$ & $W_{\lambda}$ & $v_{L S R}$ & $W_{\lambda}$ & $v_{L S R}$ & $W_{\lambda}$ & $v_{L S R}$ & $W_{\lambda}$ & $v_{L S R}$ & $W_{\lambda}$ \\
\hline 49.4 & 14 & & $<4$ & & $<5$ & 49.2 & 4: & 48.8 & 42 & 46.7 & 20 & 47.6 & 32 \\
\hline$\simeq 58$ & 2 & & $<4$ & & $<5$ & 58.5 & 14 & & $<5$ & & $<6$ & & $<6$ \\
\hline
\end{tabular}

CaII K

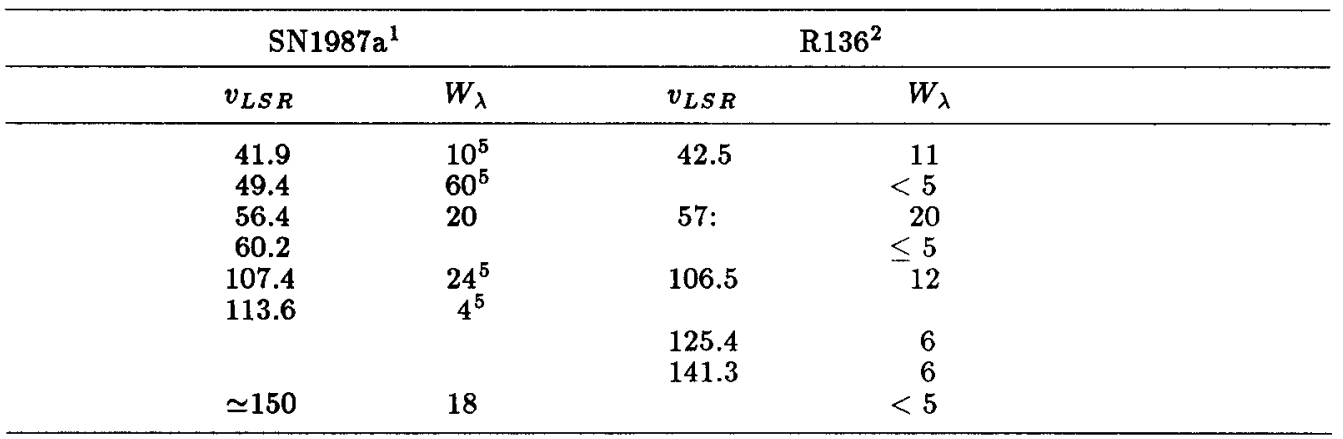

1 Vidal-Madjar et al. (1987)

2 This work

3 Molaro et al. (1989)

${ }^{4}$ Ferlet et al. (1985)

5 Blend solved from the data of Vidal-Madjar et al. (1987) 
as a broad, shallow absorption in $\mathrm{Sk}-69^{\circ} 203$, and is not detected in the other lines of sight.

In the range $+100 \mathrm{~km} \mathrm{~s}^{-1} \leq v_{L S R} \leq+150 \mathrm{~km} \mathrm{~s}^{-1}$ absorptions are detected in CaII, but not in NaI. One component at $+107 \mathrm{~km} \mathrm{~s}^{-1}$ is observed both in the supernova and, for the first time, in R136. The perfect velocity coincidence of this component along the two lines of sight suggests a common origin of the absorbing gas. If this gas were located in the LMC this would imply the existence of a coherently moving structure with linear dimensions of about $300 \mathrm{pc}$, i.e., the projected linear size corresponding to the angular separation between R136 and SN1987a at a distance of $\simeq 50 \mathrm{kpc}$. This argument favours a local origin (within a few $\mathrm{kpc}$ ) of the absorbing gas. A similar conclusion can be reached for the components at $+42 \mathrm{~km} \mathrm{~s}^{-1},+49 \mathrm{~km} \mathrm{~s}^{-1}$ and $+57 \mathrm{~km} \mathrm{~s}^{-1}$, for which a good agreement in radial velocities along the different lines of sight is also found.

Two Call components at $+125 \mathrm{~km} \mathrm{~s}^{-1}$ and $+141 \mathrm{~km} \mathrm{~s}^{-1}$, close to the detection limit, are visible toward R136 but not toward SN1987a. These features are likely associated to the absorption observed by Savage and de Boer (1981) around $v_{L S R} \simeq+130 \mathrm{~km} \mathrm{~s}^{-1}$ and again suggest a complex structure of the gas originally detected at lower resolution in the ultraviolet. Finally, a broad, shallow CaII absorption at $+150 \mathrm{~km} \mathrm{~s}^{-1}$ is present in the supernova, but not in R136.

\section{DISCUSSION AND FUTURE WORK}

In all the cases discussed above the CaII components are stronger than the corresponding $\mathrm{NaI}$ features. In many cases there are components detected in CaII, but not in NaI. These facts can be used to constrain the HI column density and the calcium to hydrogen ratio of the foreground gas, if present. The lack of detection in NaI implies $\mathrm{N}(\mathrm{NaI})<2.5 \times 10^{10} \mathrm{~cm}^{-2}$ and this in turn translates in an upper limit $\mathrm{N}(\mathrm{HI})<2 \times 10^{19} \mathrm{~cm}^{-2}$, when the ratio $\mathrm{N}(\mathrm{NaI}) / \mathrm{N}(\mathrm{HI}) \simeq 1.3 \times 10^{-9}$ given by Ferlet et al. (1985b) for the Galactic gas is used. If in the LMC the sodium is underabundant with respect to the solar value, the upper limit in the HI column density would be even higher. A cloud with hydrogen column density in the range $10^{18} \mathrm{~cm}^{-2}<\mathrm{N}(\mathrm{HI}) \leq 2 \times 10^{19} \mathrm{~cm}^{-2}$ would escape Nal detection, but not CaII detection, provided CaII depletion is very low. The same cloud would be easily detected in the ultraviolet thanks to the presence of the strongest lines of the most abundant interstellar ions in that spectral region. In fact, Savage and de Boer (1980) estimated $\mathrm{N}(\mathrm{HI})>10^{18} \mathrm{~cm}^{-2}$ from their analysis of high-velocity clouds. We conclude that the lack of $\mathrm{NaI}$ detection, even at the very low level of the data presented in this communication, is not in contraddiction with the presence of foreground halo gas of the type suggested by Savage and de Boer (1981) on the basis of their IUE data. Of course, in those directions where NaI is detected, the cloud density must be higher. The clouds must be very patchy in order to explain the strong variations of the absorptions, even between lines of sight separated only by a few arcminutes (Molaro et al. , 1989).

Recent 21-cm observations reported by de Boer et al. (1989a) indicate that high-velocity gas in the range of radial velocities of interest is present in the general direction of the LMC, but well outside the LMC boundary, in agreement with a Galactic halo origin of the observed clouds. The low HI column densities measured by de Boer et al. (1989a) - N(HI) $\leq 6 \times 10^{18} \mathrm{~cm}^{-2}-$ are consistent with the above mentioned contraints derived on the basis of our $\mathrm{NaI}$ upper limits.

A preliminary study of the kinematics and the stratification of the LMC gas is clearly important in order to address the problem of the origin of the high-velocity absorptions in that direction of the sky. Observations by means of the IUE satellite are inadequate to reach this goal because the $\mathrm{S} / \mathrm{N}$ and spectral resolution are insufficient to solve the interstellar components. A large number of lines of sight must be investigated in the optical at high spectral resolution and with sufficient $\mathrm{S} / \mathrm{N}$ in order to detect $\mathrm{NaI}$ and CaII absorptions. Perhaps the best way of tackling this problem is to survey selected regions of the Magellanic Clouds and to sample the depth structure of the gas by looking at stars distributed at different distances within each region. We plan to perform 


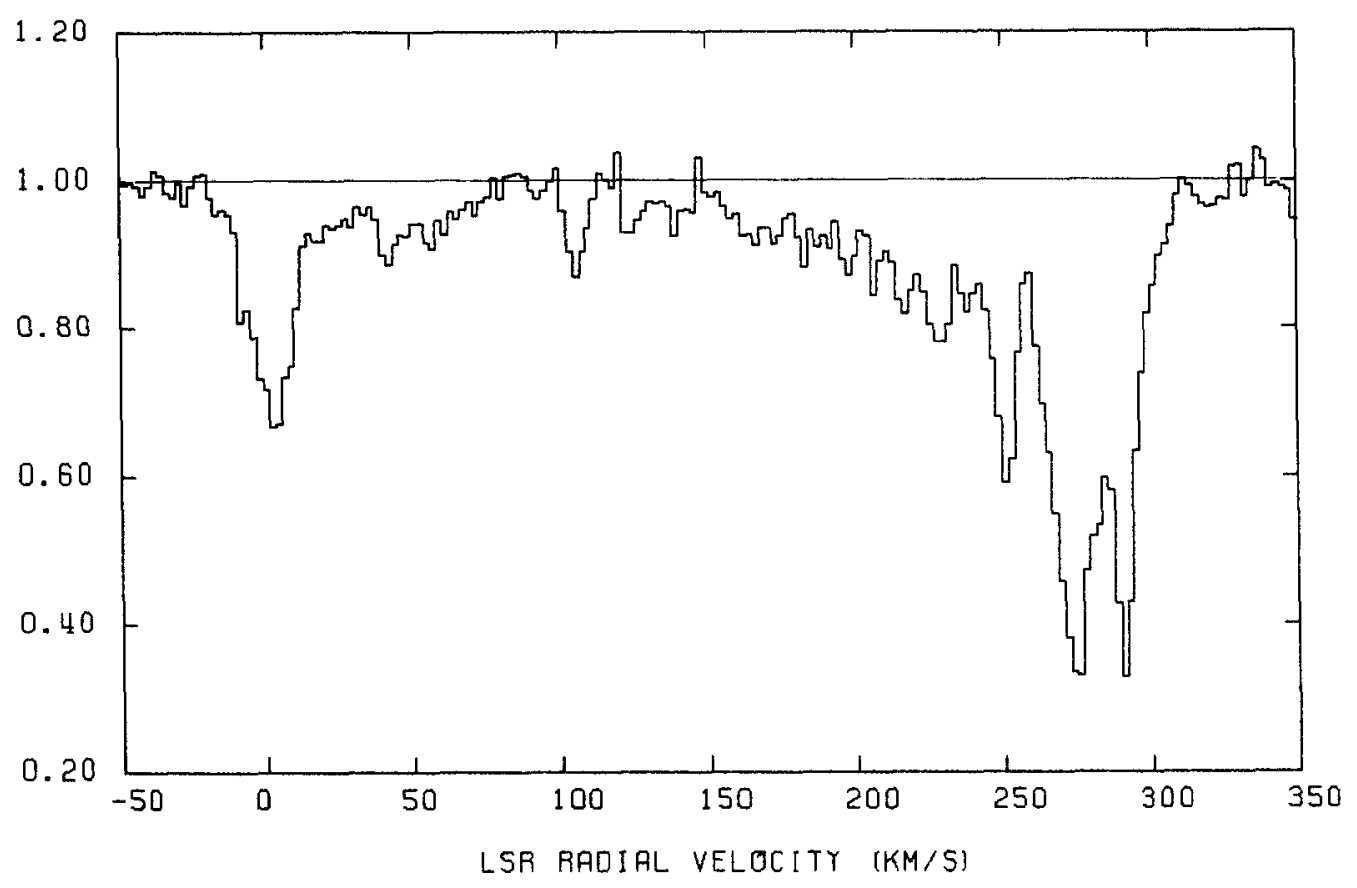

Fig. 1 - CaII K interstellar spectrum toward R136.

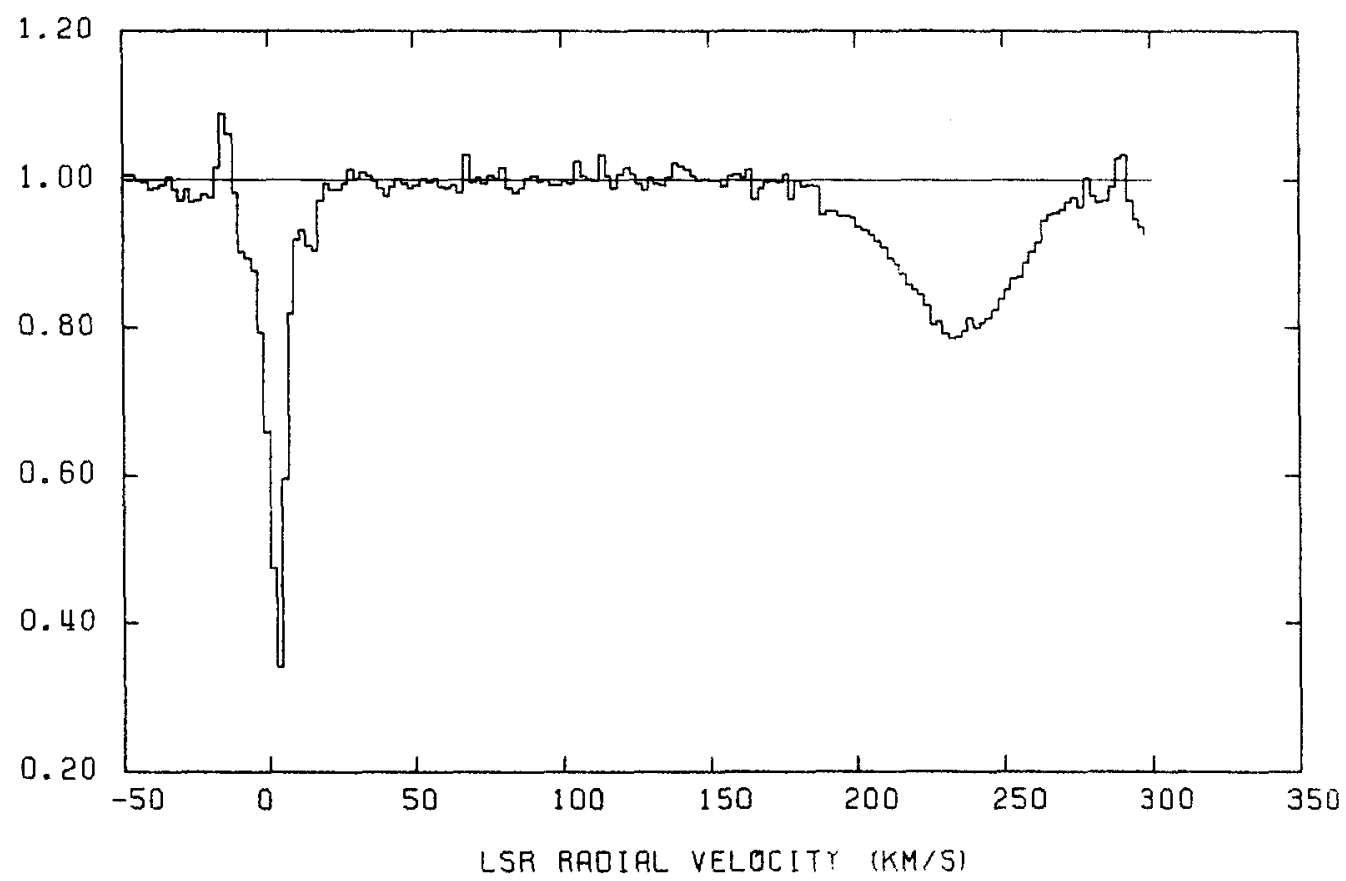

Fig. 2 - NaI D2 spectrum toward Sk-67 44 after correction for telluric contamination. The broad absorption close to $v_{L S R} \simeq 235 \mathrm{~km} \mathrm{~s}^{-1}$ is due to stellar NaI. 
these kind of observations during the next few years in the frame of a coordinated investigation of selected regions in the Magellanic Clouds (de Boer et al., 1989b). The use of a fiber link to connect the 3.6-m ESO telescope with the CES (Avila and D'Odorico, 1988) will allow to obtain $\mathrm{CaII}$ and $\mathrm{NaI}$ spectra at the required spectral resolution for a significant number of targets in the Magellanic Clouds.

\section{REFERENCES}

Avila, G., and D'Odorico,S.: 1988, Proc.of the Very Large Telescope Symposium, ed. M.H.Ulrich, (in press).

Blades, J.C., and Meaburn, J.: 1980, M.N.R.A.S., 190, 59P.

de Boer, K.S., Azzopardi, M., Baschek, B., Dennefeld, M., Israel, F.P., Molaro, P., Seggewiss, W., Spite, F., and Westerlund, B.D.: 1989b, to be pubblished on the Messenger.

de Boer, K.S., Morras, R., and Bajaja: 1989a, submitted for pubblication on Astron. Astrophys.

Ferlet, R., Dennefeld, M., and Maurice, E.: 1985a, Astron. Astrophys., 152, 151.

Ferlet, R., Vidal-Madjar, A., and Gry, C.: 1985b, Astrophys. J., 298, 838.

Molaro, P., Vladilo, G., Avila, G. and D'Odorico, S.: 1989, Astrophys. J. (Letters), 339, L63.

Savage, B.D., and de Boer, K.S.: 1979, Astrophys. J. (Letters), 230, L77.

Savage, B.D., and de Boer, K.S.: 1981, Astrophys. J., 243, 460.

Songaila, A.: 1981, Astrophys. J., 248, 945.

Songaila, A., Blades, J.C., and Hu, E.M.: 1986, Astrophys. J., 303, 198.

Vidal-Madjar, A., Andreani, P., Cristiani, S., Ferlet, R., Lanz, T., and Vladilo, G.: 1987, Astron. Astrophys., 177, L17.

\section{Discussion:}

VAN WOERDEN (Comment): From the strong variations in line strength over distances of a few arcminutes, you conclude that the lines around $+50 \mathrm{kms}^{-1}$ velocity arise in or near the Large Magellanic Cloud. However, such strong variations over small angular separations do also occur in high-velocity clouds (see following paper by B.P. Wakker) and even in low-velocity gas in the disk (as shown in Westerbok studies by Schwarz, Kalberla and Goss).

de BOER (Comment): As Hugo van Woerden just mentioned, also the high velocity gas is patchy. The problem of association of the -60 and $-130 \mathrm{kms}^{-1}$ components with the LMC is resolved. Together with Bajaja and Morras we observed in $21 \mathrm{~cm}$ directions outside the boundaries of LMC. We have found the -60 and $-130 \mathrm{kms}^{-1}$ gas outside those boundaries, so I claim these clouds belong to the Milky Way and not to the LMC. 